\title{
Graduate Students,
}

Energy, Environment \& Resource Sustainability

\section{Operational Performance and Safety Assessment of Signalized Roundabout} Jameel Hannun, Jamal Hannun, Khaled Shamiyeh, Mohammad Ghanim, Mohamed Kharbeche

\section{Introduction}

- Qatar installed traffic signals on important roundabouts to enhance operational and safety performance.

- Performance evaluation is commonly done using historical data, however to provide countermeasures in a more timely manner, simulating traffic conflicts from the observed roundabout operation is used to suggest operational and safety improvement.

\section{Objective}

- Assess safety and operational performance of a signalized roundabout in the City of Doha through the simulation approach and applying the surrogate safety assessment model

\section{Study site characteristics}

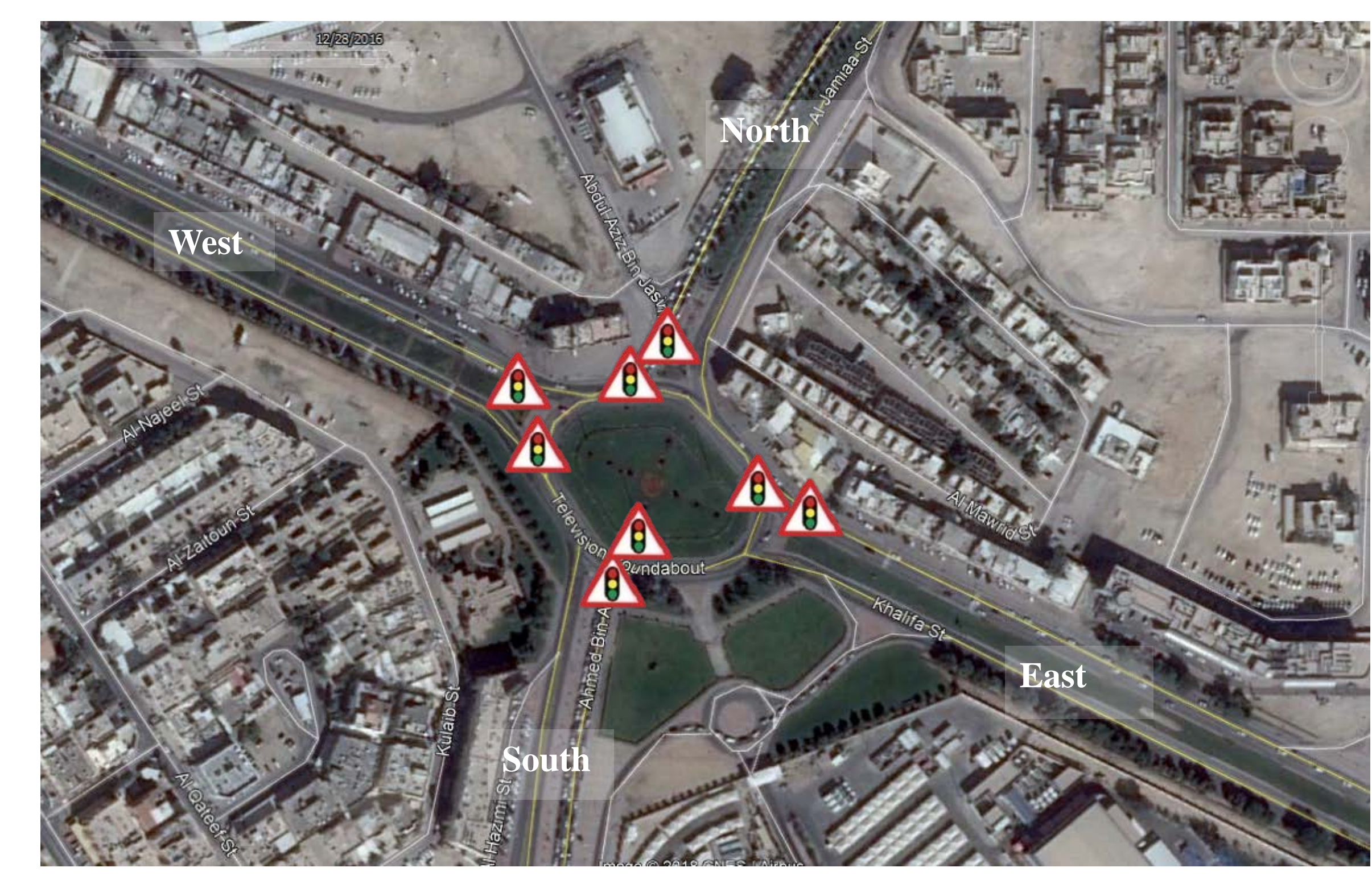

Fig. 1. The studied signalized roundabout and legs directions

\section{Traffic demand and signal timings and phasing}
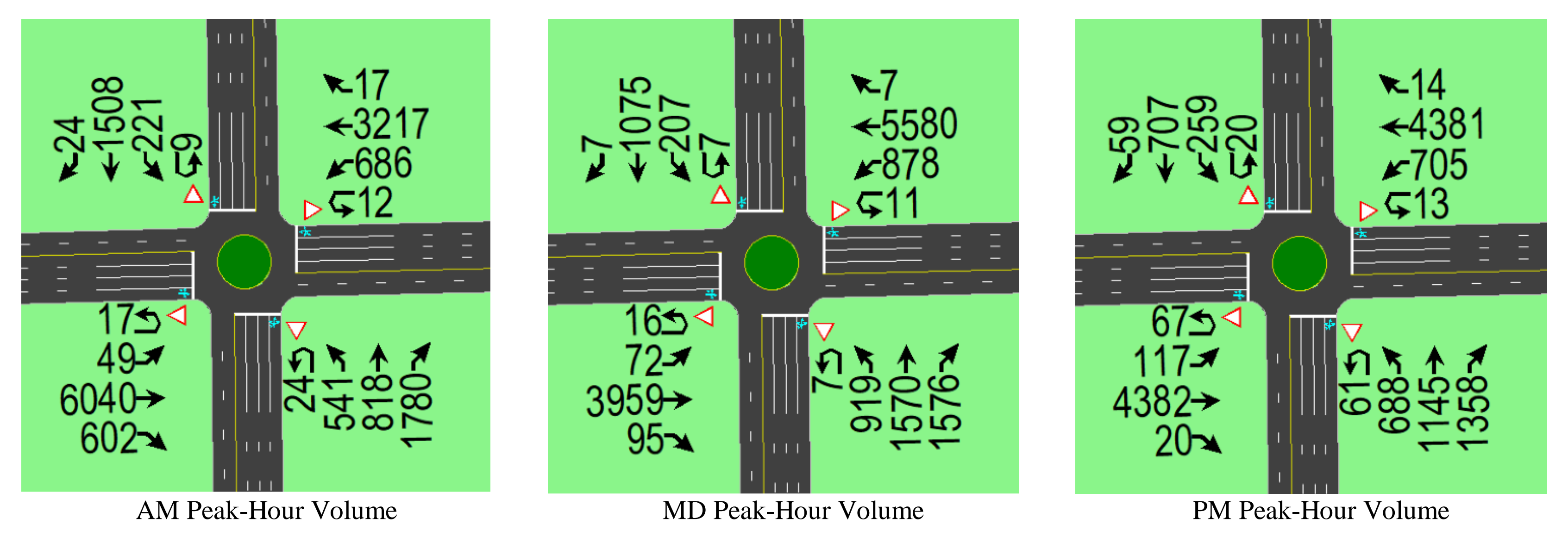

Fig. 2. Traffic Volumes for AM, MD, and PM Peak Hour

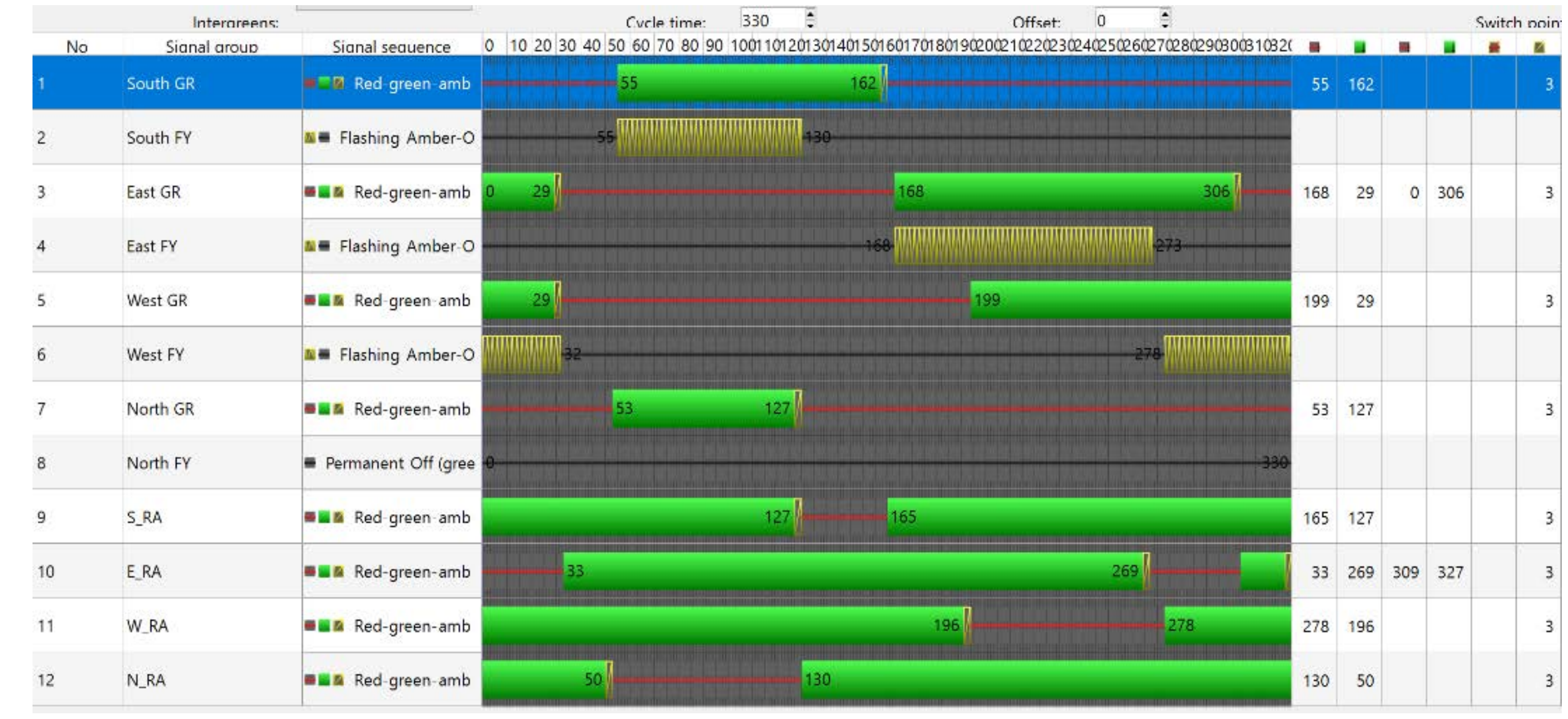

Fig. 3. Signal Timing Plans for AM Peak Hour

\section{Simulation}

Microscopic Network Modeling

- The most important step in this study is to model the signalized roundabout at the microscopic level so that operational performance data and vehicular trajectories can be collected and processed.

- VISSIM is the only microscopic traffic simulation software that is authorized by the Ministry of Transport and Communication.

\section{SSAM}

- Surrogate Safety Assessment Model (SSAM) developed by Federal Highway Administration (FHWA) is a tool that analyze vehicular trajectories to identify and categorize potential traffic conflicts. Used to determine the conflict severity and identify the type of conflict.

Table 1. Conflict severity based on Time to Collision

\begin{tabular}{|c|c|c|c|c|}
\hline Collision Risk & Extreme & High & Moderate & \\
\hline Time to Collision (TTC) & $<0.1$ & $0.1-0.5$ & $0.5-1.0$ & $1.0-1.5$ \\
\hline
\end{tabular}

\section{Results}

- Average delay is computed as the difference between the actual and the anticipated time to enter and exit the roundabout averaged across all the vehicles. Stop delay is the time spent while the vehicle is not moving.

- From Fig. 4. the PM peak hour has experienced the highest delay, and Fig. 5. show less variation for the number of stops per vehicle.

- Fig. 6. shows differences in traffic conflicts location based on the peak period. This can be explained by the fact that the signal timing plans are not properly implemented.

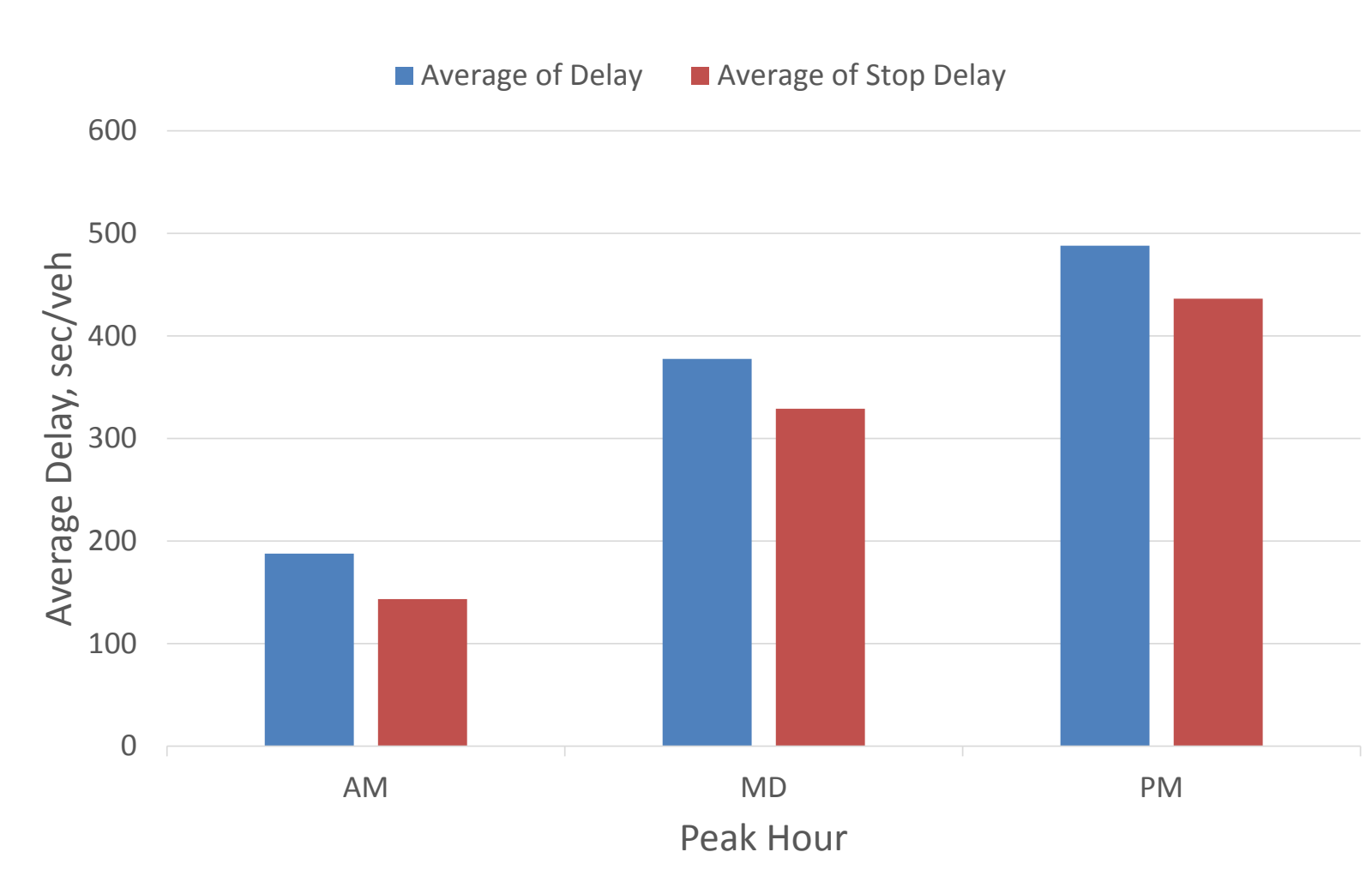

Fig. 4. Average Delay and Stop Delay for each Peak Hour Period, in sec/veh

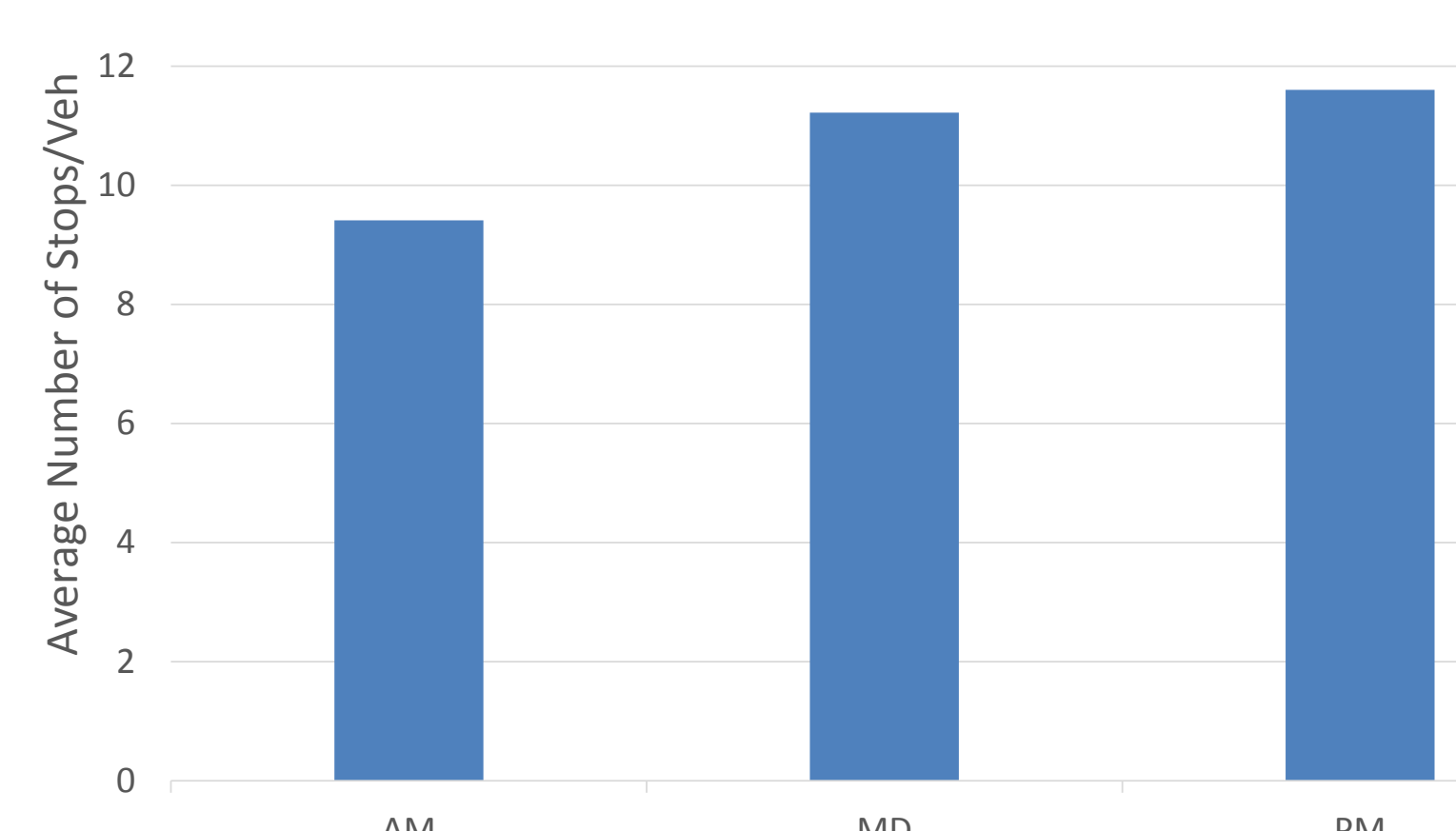

Fig. 5. Average Number of Stops per vehicle for each Peak Hour Period
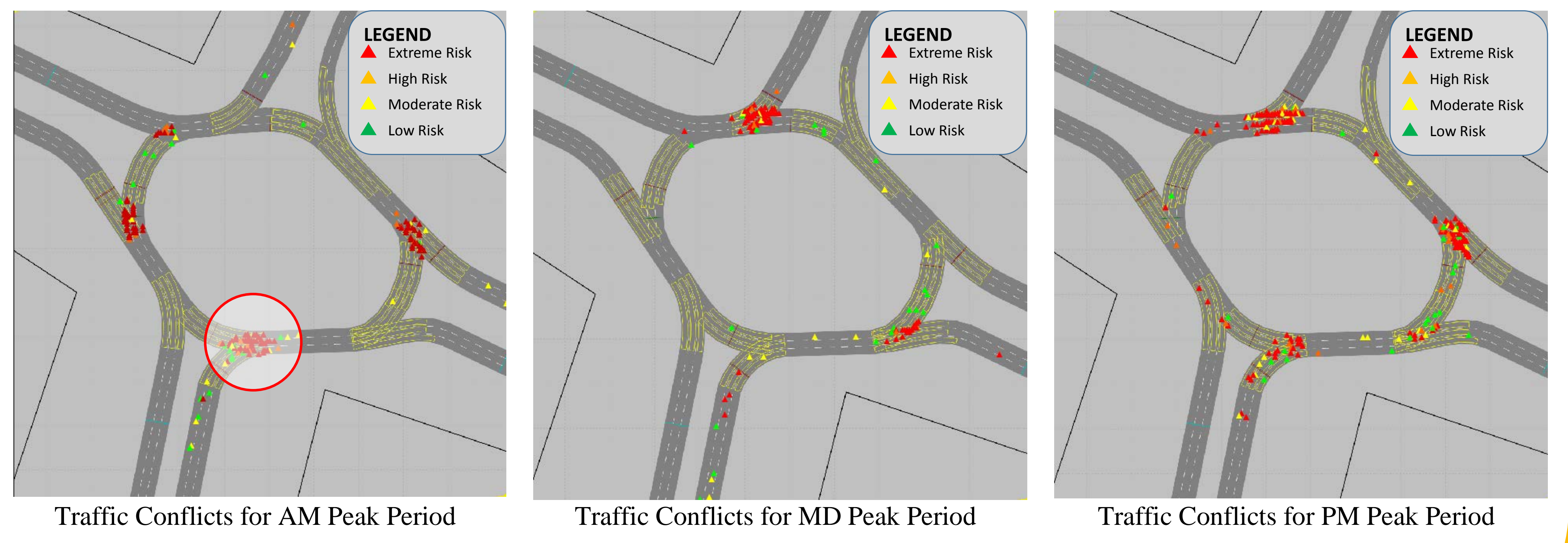

Fig. 6. Potential Location of Traffic Conflicts for AM, MD, and PM Peak hour Periods

\section{Conclusions}

- The results indicated that the signal timings and phasing schemes are associated with the level of safety at the signalized roundabout by reducing or increasing the potential traffic conflicts. Moreover, it was found that high traffic demand at signalized roundabouts is not necessarily related to the high traffic conflicts or high conflict severity.

\section{Future work}

- Expand these findings further to compare the performance of signalized roundabouts with signalized intersections.

- Investigate traffic conflicts and conflict severity for signalized roundabouts versus signalized intersections.

\section{Acknowledgements}

This publication was made possible by the UREP award (UREP19-038-2-015) from the Qatar National Research Fund (a member of The Qatar Foundation). The statements made herein are solely the responsibility of the authors. 